\title{
Erratum: Identification of the Mott Insulating Charge Density Wave State in $1 T-\mathrm{TaS}_{2}$ [Phys. Rev. Lett. 126, 196406 (2021)]
}

Dongbin Shin, Nicolas Tancogne-Dejean, Jin Zhang, Mahmut Sait Okyay, Angel Rubio, and Noejung Park®

(Received 18 November 2021; published 12 January 2022)

DOI: 10.1103/PhysRevLett.128.029902

We wrote in our Letter that "On the other hand, recent scanning tunneling microscopy and transmission electron microscopy revealed that the Ta atoms in different layers are in good order along the out-of-plane direction [1], and such type of double layer order is not likely in practice [2]." Here, we correct the citations as "Recent scanning tunneling microscopy and transmission electron microscopy studies have revealed various stacking sequences, such as $A, L$, and other configurations, and the nature of the band gap [1,3], while indications of the dimerized double-layer order were also provided in the experiments of scanning tunneling microscopy and low energy electron diffraction [2,4,5]." As our Letter is mainly focused on the Coulomb effect in variously stacked $\mathrm{TaS}_{2}$ layers, this change does not affect the main result. Additionally, the label of the vertical axis of Fig. S2, in the Supplemental Material, needs to be modified as $\mathrm{E}_{U}(\mathrm{Ry})$ to make better consistency with Eq. (1) in the main text.

[1] R. Hovden, A. W. Tsen, P. Liu, B. H. Savitzky, I. El Baggari, Y. Liu, W. Lu, Y. Sun, P. Kim, A. N. Pasupathy, and L. F. Kourkoutis, Proc. Natl. Acad. Sci. U.S.A. 113, 11420 (2016).

[2] C. J. Butler, M. Yoshida, T. Hanaguri, and Y. Iwasa, Nat. Commun. 11, 2477 (2020).

[3] Z. Wu, K. Bu, W. Zhang, Y. Fei, Y. Zheng, J. Gao, X. Luo, Z. Liu, Y. ping Sun, and Y. Yin, arXiv:2105.08663.

[4] G. von Witte, T. Kißlinger, J. G. Horstmann, K. Rossnagel, M. A. Schneider, C. Ropers, and L. Hammer, Phys. Rev. B 100, 155407 (2019).

[5] S. H. Lee, J. S. Goh, and D. Cho, Phys. Rev. Lett. 122, 106404 (2019). 